\title{
Factores asociados al fracaso de la terapia de conductos radiculares
}

\section{Factors associated to the failure of the root canal therapy}

\section{Resumen}

Objetivo: Identificar los principales factores asociados al fracaso en el tratamiento pulpo radicular. Métodos: Se realizó un estudio relacional longitudinal prospectivo en la localidad de Santa Clara, Cuba, en el periodo 2015-2017 donde se incluyó a 448 pacientes. Las variables estudiadas con relación al fracaso de la terapia fueron: edad, género, antecedentes sistémicos, apertura bucal limitada, grupo y localización de los dientes, anomalías dentarias de espacio, morfología radicular, alteración del número de raíces, canales reducidos, bifurcación del canal, calcificaciones pulpares, sensibilidad pulpar, reabsorción, complejidad del diagnóstico, dolor o inflamación preoperatorio, interpretación radiográfica, acceso cameral con complicaciones, dolor durante el tratamiento, calidad de la obturación y enfermedad periodontal. Resultados: De los 31 casos que presentaron el tamaño del canal reducido, 17 fracasaron, siendo este el factor de mayor significación en relación al fracaso endodóntico $(p<0,01)$. Otras asociaciones significativas se encontraron en la sensibilidad pulpar que representó el $17 \%$ de los dientes $(p=0,001)$, morfología radicular alterada observada en el $58 \%$ de los casos $(p=0,005)$, y la calidad de la obturación deficiente del $4,9 \%$ de estos $(p=0,009)$. Conclusiones: El estudio evidenció una asociación significativa de varios factores con la evolución de la terapia; registrándose la edad, grupo de dientes, localización de los dientes, morfología radicular, tamańo de los conductos, sensibilidad pulpar, calidad de la obturación y la enfermedad periodontal como los de mayor compromiso.

Palabras clave: Atención odontológica; Endodoncia; Tratamiento del conducto radicular.
ISSN: 1560-9111

\section{Artículo Original}

Lilian Toledo Reyes ${ }^{1, a}$, Amarilys Labrada Benítez 2,b, Rafael Valdés Álvarez 1,c

\author{
${ }^{1}$ Facultad de Estomatología. Universidad de \\ Ciencias Médicas. Villa Clara, Cuba. \\ ${ }^{2}$ Policlínico Martha Abreu. Villa Clara. Cuba. \\ a Especialista de II Grado en Estomatología \\ General Integral. Máster en Salud Bucal Comu- \\ nitaria.

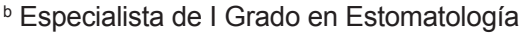 \\ General Integral.
}

c Especialista de II Grado en Estomatología General Integral. Doctor en Ciencias Médicas.

Correspondencia: Lilian Toledo Reyes

Correo electrónico: liliantr@infomed.sld.cu Universidad de Ciencias Médicas de Villa Clara. Facultad de Estomatología. Departamento de Estomatología Integral. Carretera de Acueducto y Circunvalación Sur. Villa Clara, Cuba.

\section{Coautores:}

Amarilys Labrada Benítez

amarilyslb@nauta.cu

Rafael Valdés Álvarez

rafvald@infomed.sld.cu

Conflicto de intereses: Los autores declaran no tener conflictos de interés.

Fuente de financiamiento: Autofinanciado

Fecha de recepción: 04/03/18

Fecha de aceptación: 28/04/18

\begin{abstract}
Objective: To identify the main factors associated to the failure of endodontic therapy. Methods: A relational prospective longitudinal study was performed in the city of Santa Clara in the period 2015-2017; resulting in a study group consisted of 448 patients. The selected variables associated with the failure of therapy were: age, gender, medical history, mouth opening, group location and position of tooth, root morphology, root apex number alteration root, visibility and size of the canals, branch, calcifications, pulp condition, reabsorption, diagnosis complexity, pain or swelling before therapy, difficulty radiographic diagnosis, cameral access complexity, pain during the treatment, quality of canal filling and periodontal disease. Results: Of 31 cases with reduced root canal size, 17 fail, being this the factor of greater significance $(\mathrm{p}<0.01)$ related to the therapy failure. Other factors with strong association were pulp condition $(\mathrm{p}=0.001)$, root morphology $(\mathrm{p}=0.005)$, as well as the quality of canal filling $(\mathrm{p}=0.009)$ which represent the $17 \%$, $58 \%$ and $4.9 \%$ of the observed cases respectively. Conclusions: Age, group and location of tooth, root morphology, size of the canals, pulp condition, quality of filling and periodontal disease were the main factors associated to the root canal treatment failure.
\end{abstract}

Keywords: Dental care; Endodontics; Root canal therapy. 


\section{Introducción}

Los recientes avances tecnológicos han permitido desarrollar opciones terapéuticas que se utilizan para tratar dientes con pronóstico difícil ${ }^{1}$. La mayoría de los autores coinciden en que la permanencia del diente es mejor que su pérdida y utilizan el tratamiento endodóntico como una medida de saneamiento, evitando así el desarrollo de nuevas lesiones. El objetivo de la terapia endodóntica es la prevención y/o el tratamiento de las alteraciones pulpares y de sus posibles repercusiones sobre los tejidos periapicales. El tratamiento endodóntico conservador (no quirúrgico) es común en la práctica odontológica; es por eso que es imperativo conocer los factores asociados a su resultado final.

Se reporta en la literatura tasas de éxito respecto al tratamiento entre un 86 y $95 \%{ }^{2}$. Apuntes menos alentadores señalan la incidencia o frecuencia de fracaso durante la terapia de canales entre un 25 a un $40 \%$ de los $\operatorname{casos}^{3}$. Este se vincula generalmente a un diagnóstico incorrecto, inadecuada selección del caso, errores en el plan de tratamiento y una técnica operatoria deficiente ${ }^{4}$. Algunas veces, las fallas se producen a pesar de la adherencia rígida a los principios básicos del proceder.

Históricamente el desenlace de la terapia se ha juzgado en relación a la posibilidad de la esterilización del sistema de canales radiculares combinada con la necesidad de obtener un sellado apical hermético ${ }^{1}$.

En el contexto internacional se han brindado varias clasificaciones de los factores que influyen en el resultado de la terapia y se menciona la condición pulpo periapical previa, compleja anatomía del diente y sistema de canales, así como complicaciones durante el procedimiento, entre otros aspectos, como los de mayor compromiso ${ }^{4-6}$.

Como otros parámetros relacionados con la evolución desfavorable del tratamiento los reportes señalan: la posición en el arco, dientes con curvatura radicular extrema, acceso cameral previo con complicaciones y el factor visibilidad y tamaño de los canales ${ }^{1,6,7}$. Algunas condicionantes están asociadas al estado sistémico del paciente y también deben ser tenidas en cuenta.

La evaluación precisa de la posibilidad de fracaso de la terapia endodóntica se torna difícil al valorarse muchas variables que pudieran complejizar el caso. Esto puede afectar la capacidad del profesional de asumir un pronóstico con objetividad, sobre todo si se trata de un profesional joven. El formulario de determinación de la dificultad del caso endodóntico propuesto por la Asociación Americana de Endodoncia (AAE) agrupa varias categorías de estas variables y es usado con el fin de perfeccionar la evaluación del pronóstico en la terapia endodóntica ${ }^{1}$.

Teniendo en cuenta lo antes expuesto y considerando la importancia que tiene el tratamiento pulpo radicular en la práctica cotidiana para preservar el órgano dentario, se realiza esta investigación con el objetivo de identificar los principales factores asociados al fracaso del tratamiento en la población objeto de estudio.

\section{Métodos}

Se realizó un estudio relacional, longitudinal y prospectivo, en Santa Clara, Cuba, en el período comprendido de octubre de 2015 a enero de 2017.

La población incluyó 1104 tratamientos pulpo radiculares llevados a cabo en el periodo de tiempo seleccionado en la localidad. La muestra quedó conformada por 448 dientes. Para la selección de la muestra se consideró que la estimación de la proporción de fracasos contenidos en la misma estuviese acotada con un margen de error (e) de por lo menos $\pm 5 \%$ para un intervalo de confianza de al menos el 95\%. Para garantizar lo anterior, la muestra debió cumplir con el siguiente criterio: $\mathrm{n}=\mathrm{Z}^{2} \cdot \mathrm{p} \cdot \mathrm{q} / \mathrm{e}^{2}$, donde $\mathrm{Z}$ en este caso se tomó igual a 2 para garantizar un intervalo de confianza ligeramente superior al 95\%, p y q se hicieron igual a 0,5, lo que equivale a contemplar la mayor varianza posible de la distribución muestral. Sustituyendo estos valores en la expresión anterior se seleccionó valor de $\mathrm{n}$ tal que: $\mathrm{n} \geq 1$ / $e^{2}=1 /(0,05)^{2}=400$.

Se utilizó un muestreo por conglomerados bietápico, escogiéndose en la primera etapa el $50 \%$ de los conglomerados, dados por las unidades de salud que prestaron servicios estomatológicos en la ciudad de Santa Clara. En la segunda etapa, para cada uno de los conglomerados resultantes, se seleccionó una parte de los tratamientos endodónticos realizados durante el primer semestre del 2015, de forma tal que fuese proporcional al tamaño de los mismos hasta satisfacer el criterio de tamaño de la muestra $(n \geq 400)$.

Selección de las variables. Las variables asociadas al fracaso del tratamiento endodóntico fueron definidas a partir de la revisión exhaustiva de la literatura sobre el tema. La búsqueda bibliográfica se realizó a través de bases de datos electrónicas (EBSCO, Cochrane, MEDLINE y LILACS), utilizando motores de búsqueda (Pubmed y DeCS); se incluyeron artículos en inglés y español. De acuerdo a los descriptores definidos atendiendo al tema de interés para la publicación fueron seleccionadas 36 referencias de 126 consultadas. Las mismas correspondientes a artículos originales, trabajos de diploma y revisiones de literatura, quedando excluidos los reportes de casos.

El formulario elaborado para la recolección de la información se sustentó en los criterios de la Asociación Americana de Endodoncia (AAE) ${ }^{8}$ con respecto a parámetros de complejidad de la terapia endodóntica. Por lo tanto es una adaptación de las prácticas internacionales al escenario de estudio, para lo cual se tuvo en cuenta el criterio de expertos. La selección de expertos fue llevada a cabo a través del método Delphi; considerando para la selección de los mismos el nivel de conocimientos, categoría científica y publicaciones de estos en relación al tema objeto de estudio.

Las variables incluidas en la investigación fueron las siguientes: edad, género, antecedentes sistémicos y apertura bucal limitada. Se identificaron también el grupo de dientes (anteriores o posteriores) y localización de los 
mismos (maxilares y mandibulares), anomalías dentarias de espacio (según la presencia de alteraciones de posición, dirección, de volumen o forma que impidieran el acceso cameral del diente y el paso libre del instrumental al canal), morfología radicular a partir de la presencia de curvatura radicular medida en grados con un semicírculo sobre la radiografía empleándose el método de Schneider ${ }^{9}$, alteración del número de raíces (hallazgo radiográfico de un diente con más raíces de lo anatómicamente descrito como común para ese grupo dentario), canal reducido (en correspondencia con la imagen radiográfica y exploración inicial del mismo con limas de la subserie 0,6-0,8-10, de acuerdo al criterio del profesional), bifurcación del canal (hallazgo radiográfico de un canal que se bifurca en algún tercio radicular) y presencia de calcificaciones pulpares (observable radiográficamente). Con respecto a los aspectos clínicos y relacionados con el diagnóstico se registraron: sensibilidad pulpar, reabsorción, complejidad del diagnóstico (cuando los signos y síntomas conducían a un amplio diagnóstico diferencial), dolor o inflamación preoperatorio (referido por el paciente en la semana antes de iniciar la terapia) e interpretación radiográfica (considerando la interpretación radiográfica que no ofrece dificultades como sencilla o compleja cuando existía superposición de estructuras, o la calidad de la radiografía realizada era inadecuada).

En relación al tratamiento, las variables observadas fueron: dolor durante el tratamiento, acceso cameral con complicaciones (falsas vías, perforaciones, apertura sub-extendida o sobre-extendida), calidad de la obturación del conducto (correcto o incorrecto valorando el sellado hermético del conducto radiográficamente), presencia de periodontitis crónica (cuando existía presencia de sacos periodontales y evidencia radiográfica de cualquier patrón de destrucción ósea). Fue registrado además el resultado del tratamiento de conductos en relación al éxito o fracaso de la terapia a través de hallazgos clínicos y radiográficos concernientes, utilizando criterios de Gutmann ${ }^{10}$. Dentro de los aspectos clínicos asociados al fracaso se tuvo en cuenta la presencia de síntomas subjetivos persistentes, trayecto fistuloso o inflamación recurrentes, molestia a la percusión y palpación, evidencia de fractura dentaria irreparable, movilidad excesiva o deterioro periodontal progresivo e incompetencia funcional del diente afectado. Como parámetros radiográficos fue valorada la presencia de ensanchamiento del ligamento periodontal más de 2 milímetros, la ausencia de reparación ósea en la zona radiolúcida y/o aumento de tamaño de la misma, la ausencia de formación de una nueva lámina dura o aumento significativo en la densidad ósea en los tejidos perirradiculares y la existencia de radiolucidez perirradicular ósea nueva incluyendo áreas de rarefacción laterales.

Recolección de la información. Para lograr la recolección de datos se empleó la anamnesis, examen clínico extra e intrabucal con el auxilio del sillón dental, espejo bucal plano, pinzas, explorador número 5 , sonda periodontal milimetrada y luz artificial, y el examen radiográfico, mediante radiografías periapicales.
La recolección de datos y los tratamientos fueron realizados por estomatólogos generales que brindaron servicio en las unidades asistenciales del sistema público. Antes de iniciar la investigación fueron homogenizados criterios en relación al protocolo del tratamiento y calibrados inicialmente los profesionales a cargo de la recolección de los datos.

Durante la primera cita se registraron las variables correspondientes a factores asociados al paciente, factores anatómicos, factores clínicos relacionados con la terapia, así como otros factores de interés mediante un formulario desarrollado a partir de criterios de la AAE.

Para realizar el procedimiento en relación a la terapia endodóntica se realizó el aislamiento del campo operatorio, limpieza del diente con clorhexidina al $0,2 \%$, acceso cameral y exploración del canal con limas de la subserie $(0,6-0,8-10)$. La longitud de trabajo fue medida mediante la técnica radiográfica usando películas (Ektaspeed Plus Kodak talla 2) y equipo de RX $127 \mathrm{v}-70 \mathrm{kv}-8 \mathrm{~mA}$ (Dabi Atlante modelo Spectro 70x). El procedimiento radiográfico fue hecho por el método tiempo/temperatura utilizándose soluciones reveladoras y fijadoras Kodak. El análisis de las radiografías fue realizando con auxilio del negatoscopio. A partir de la estimación de la longitud aparente inicial tomada con regla milimetrada sobre una radiografía preoperatoria, se le restaron dos milímetros a la medida de la lima como margen de seguridad y con los topes de goma ajustados a esta nueva medición se avanzó en los canales hasta alcanzar la constricción apical y fue tomada otra radiografía, para dientes multiradiculares fue realizada una proyección ortorradial y otra disto o mesiorradial desplazando el cono en uno u otro sentido unos $20^{\circ} \mathrm{o}$ $30^{\circ}$, para logar la mejor visibilidad de los instrumentos en el interior del canal. La longitud definitiva fue establecida a 1 o 2 milímetros del ápice radiográfico. Se utilizaron limas Hedstrom H-Files (IMD ${ }^{\oplus}$, China) en la preparación biomecánica la cual se llevó a cabo de forma manual y se irrigó con hipoclorito de sodio al 2,5\%, empleándose puntas de papel absorbente para secar el canal. Fue utilizado el Dentofar (EuFar S.A. ๑, Colombia), como medicación intracanal en casos de necrosis y área de rarefacción apical. El procedimiento fue realizado en tres sesiones. En la tercera sesión se realizó la obturación de conductos empleando la técnica de condensación lateral, usando gutapercha y sellador endodóntico GrossFar (EuFar S.A. ๑, Colombia).

La recolección de la información fue completada en la cita para la obturación de conducto y posteriormente se examinaron los pacientes a los dos años, donde se valoró el éxito o fracaso del tratamiento endodóntico mediante el registro de parámetros clínicos y radiográficos.

Técnicas de procesamiento y análisis. La información resultante se presentó en tablas y figuras donde se muestran frecuencias absolutas y porcentajes. En el análisis bivariado para evaluar la presencia de asociación estadística entre las variables y el criterio de referencia (resultado final de la terapia de conductos), se empleó la significación asociada al estadígrafo $\chi^{2}$. De acuerdo 
con el valor de $p$, fue clasificada la asociación en: muy significativa: si $p$ era menor que 0,01 ; significativa: si $p$ era mayor o igual que 0,01 y menor que 0,05 ; no significativa: si $p$ era mayor o igual que $0,05{ }^{11}$.

Aspectos éticos. Se tuvieron en cuenta los reglamentos éticos para la investigación en humanos, por lo que se solicitó el consentimiento a pacientes y a la Dirección Municipal de Estomatología de Santa Clara, con la finalidad de obtener la colaboración y anuencia de estos quedando esta expresada de forma oral y escrita. Se explicaron los fines de la investigación, y se aclaró que el estudio no implicaría daño físico ni moral, y que se respetaría la autonomía de los pacientes en caso de decidir abandonar el estudio.

\section{Resultados}

De los 448 tratamientos de canales radiculares realizados en la población objeto de estudio, 60 fracasaron. Esto equivale a un $13,39 \%$ de fracaso contra un $86,61 \%$ de éxito en la terapia de canales como se observa en la Figura 1.

En la tabla 1 se refleja el comportamiento de los factores relacionados con el paciente presumiblemente asociados al resultado de la terapia en la muestra seleccionada. Se presentan para el sexo femenino un total de 31 fracasos en el tratamiento y en el masculino 29 casos de fracasos. Con una asociación significativa con el resultado de la terapia se observa la edad $(p=0,012)$. La mayor proporción de fracasos se encuentra en tratamientos practicados a pacientes mayores de 60 años (de 61 tratamientos ejecutados en este grupo, 16 fracasaron y 45 tuvieron éxito, durante el periodo de tiempo evaluado).

La tabla 2 registra una asociación significativa $(0,01 \leq p$ $<0,05)$, con un nivel de confianza del $95 \%$ para las variables grupo de dientes $(p=0,015)$ y localización de los dientes $(p=0,040)$. Mientras que la morfología radicular $(p=0,005)$ y el tamaño del canal $(p<0,001)$ mostraron una asociación muy significativa $(p<0,01)$ con respecto al fracaso de la terapia.

También la sensibilidad pulpar muestra una asociación muy significativa $(p=0,001)$ según se observa en la tabla 3.

Otro hallazgo muy significativo se observa en relación a la calidad de la obturación $(p=0,009)$ en la tabla 4 donde siete de los 22 tratamientos con deficiente calidad en el relleno fracasaron.

La presencia de enfermedad periodontal registrada en la tabla $5(p=0,01)$ tuvo un valor significativo con respecto al fracaso del tratamiento.

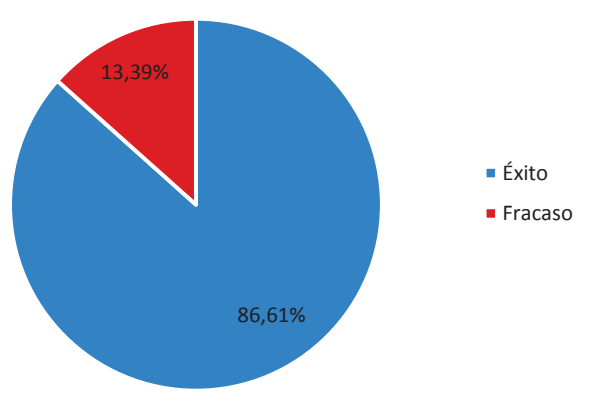

Figura 1. Resultados de la terapia pulpo radicular en la población objeto de estudio

Tabla 1. Factores relacionados con los pacientes asociados al resultado de la terapia

\begin{tabular}{|c|c|c|c|c|c|c|c|c|c|}
\hline \multirow{3}{*}{ Variable } & & \multicolumn{4}{|c|}{ Resultado } & \multirow{2}{*}{\multicolumn{2}{|c|}{ Total }} & \multirow{3}{*}{ Chi } & \multirow{3}{*}{$\mathrm{p}$} \\
\hline & & \multicolumn{2}{|c|}{ Éxito } & \multicolumn{2}{|c|}{ Fracaso } & & & & \\
\hline & & $\mathbf{n}$ & $\%$ & $\mathbf{n}$ & $\%$ & $\mathbf{n}$ & $\%$ & & \\
\hline \multirow{2}{*}{ Género } & Femenino & 212 & 47,3 & 31 & 6,9 & 243 & 54,2 & \multirow{2}{*}{0,184} & \multirow{2}{*}{0,667} \\
\hline & Masculino & 176 & 39,3 & 29 & 6,5 & 205 & 45,8 & & \\
\hline \multirow{4}{*}{ Edad (años) } & $12-19$ & 44 & 9,8 & 4 & 0,9 & 48 & 10,7 & \multirow{4}{*}{10,838} & \multirow{4}{*}{0,012} \\
\hline & $20-34$ & 126 & 28,1 & 19 & 4,2 & 145 & 32,4 & & \\
\hline & $35-59$ & 173 & 38,6 & 21 & 4,7 & 194 & 43,3 & & \\
\hline & 60 o más & 45 & 10,0 & 16 & 3,6 & 61 & 13,6 & & \\
\hline \multirow{2}{*}{$\begin{array}{l}\text { Antecedentes } \\
\text { Sistémicos }\end{array}$} & Sí & 76 & 17,0 & 16 & 3,6 & 92 & 20,5 & \multirow{2}{*}{1,595} & \multirow{2}{*}{0,206} \\
\hline & No & 312 & 69,6 & 44 & 9,8 & 356 & 79,5 & & \\
\hline \multirow{2}{*}{$\begin{array}{l}\text { Apertura bucal } \\
\text { Limitada }\end{array}$} & Sí & 2 & 0,4 & 1 & 0,2 & 3 & 0,7 & \multirow{2}{*}{1,035} & \multirow{2}{*}{0,308} \\
\hline & No & 386 & 86,2 & 59 & 13,2 & 445 & 99,3 & & \\
\hline
\end{tabular}


Tabla 2. Factores anatómicos asociados al resultado de la terapia

\begin{tabular}{|c|c|c|c|c|c|c|c|c|c|}
\hline \multirow{3}{*}{ Variable } & & \multicolumn{4}{|c|}{ Resultado } & \multirow{2}{*}{\multicolumn{2}{|c|}{ Total }} & \multirow{3}{*}{ Chi } & \multirow{3}{*}{$\mathrm{p}$} \\
\hline & & \multicolumn{2}{|c|}{ Éxito } & \multicolumn{2}{|c|}{ Fracaso } & & & & \\
\hline & & $\mathbf{n}$ & $\%$ & $\mathbf{n}$ & $\%$ & $\mathbf{n}$ & $\%$ & & \\
\hline \multirow{2}{*}{ Grupo de diente } & Posterior & 92 & 20,5 & 23 & 5,1 & 115 & 25,7 & \multirow{2}{*}{5,822} & \multirow{2}{*}{0,015} \\
\hline & Anterior & 296 & 66,1 & 37 & 8,3 & 333 & 74,3 & & \\
\hline \multirow{2}{*}{$\begin{array}{l}\text { Localización de los } \\
\text { dientes }\end{array}$} & Mandíbula & 67 & 15,0 & 17 & 3,8 & 84 & 18,8 & \multirow{2}{*}{4,176} & \multirow{2}{*}{0,040} \\
\hline & Maxilar & 321 & 71,7 & 43 & 9,6 & 364 & 81,3 & & \\
\hline \multirow{2}{*}{$\begin{array}{l}\text { Anomalía dentarias } \\
\text { de espacio }\end{array}$} & Sí & 11 & 2,5 & 4 & 0,9 & 15 & 3,3 & \multirow{2}{*}{2,357} & \multirow{2}{*}{0,124} \\
\hline & No & 377 & 84,2 & 56 & 12,5 & 433 & 96,7 & & \\
\hline \multirow{2}{*}{ Morfología radicular } & Sí & 153 & 34,2 & 35 & 7,8 & 188 & 42,0 & \multirow{2}{*}{7,622} & \multirow{2}{*}{0,005} \\
\hline & No & 235 & 52,5 & 25 & 5,6 & 260 & 58,0 & & \\
\hline \multirow{2}{*}{$\begin{array}{l}\text { Alteración del número de } \\
\text { raíces }\end{array}$} & Sí & 18 & 4,0 & 6 & 1,3 & 24 & 5,4 & \multirow{2}{*}{2,945} & \multirow{2}{*}{0,086} \\
\hline & No & 370 & 82,6 & 54 & 12,1 & 424 & 94,6 & & \\
\hline \multirow{2}{*}{$\begin{array}{l}\text { Bifurcación del } \\
\text { conducto }\end{array}$} & Sí & 5 & 1,1 & 1 & 0,2 & 6 & 1,3 & \multirow{2}{*}{0,0561} & \multirow{2}{*}{0,812} \\
\hline & No & 383 & 85,5 & 59 & 13,2 & 442 & 98,7 & & \\
\hline \multirow{2}{*}{ Tamaño del canal } & Reducido & 14 & 3,1 & 17 & 3,8 & 31 & 6,9 & \multirow{2}{*}{49,321} & \multirow{2}{*}{$<0,001$} \\
\hline & No Reducido & 374 & 83,5 & 43 & 9,6 & 417 & 93,1 & & \\
\hline \multirow{2}{*}{$\begin{array}{l}\text { Presencia de } \\
\text { calcificaciones pulpares }\end{array}$} & Sí & 10 & 2,2 & 3 & 0,7 & 13 & 2,9 & \multirow{2}{*}{1,082} & \multirow{2}{*}{0,298} \\
\hline & No & 378 & 84,4 & 57 & 12,7 & 435 & 97,1 & & \\
\hline
\end{tabular}

Tabla 3. Factores diagnósticos y clínicos asociados al resultado de la terapia

\begin{tabular}{|c|c|c|c|c|c|c|c|c|c|}
\hline \multirow{3}{*}{ Variable } & & \multicolumn{4}{|c|}{ Resultado } & \multirow{2}{*}{\multicolumn{2}{|c|}{ Total }} & \multirow{3}{*}{ Chi } & \multirow{3}{*}{$\mathbf{p}$} \\
\hline & & \multicolumn{2}{|c|}{ Éxito } & \multicolumn{2}{|c|}{ Fracaso } & & & & \\
\hline & & $\mathrm{n}$ & $\%$ & $\mathrm{n}$ & $\%$ & $\mathbf{n}$ & $\%$ & & \\
\hline \multirow{2}{*}{ Sensibilidad pulpar } & Sí & 57 & 12,7 & 19 & 4,2 & 76 & 17,0 & \multirow{2}{*}{10,630} & \multirow{2}{*}{0,001} \\
\hline & No & 331 & 73,9 & 41 & 9,2 & 372 & 83,0 & & \\
\hline \multirow{2}{*}{ Reabsorción } & Sí & 5 & 1,1 & 0 & 0,0 & 5 & 1,1 & \multirow{2}{*}{0,7819} & \multirow{2}{*}{0,376} \\
\hline & No & 383 & 85,5 & 60 & 13,4 & 443 & 98,9 & & \\
\hline \multirow{2}{*}{$\begin{array}{l}\text { Complejidad del } \\
\text { diagnóstico }\end{array}$} & Sí & 4 & 0,9 & 1 & 0,2 & 5 & 1,1 & \multirow{2}{*}{0,1900} & \multirow{2}{*}{0,662} \\
\hline & No & 384 & 85,7 & 59 & 13,2 & 443 & 98,9 & & \\
\hline \multirow{2}{*}{$\begin{array}{l}\text { Dolor o inflamación } \\
\text { preoperatorio }\end{array}$} & Sí & 50 & 11,2 & 12 & 2,7 & 62 & 13,8 & \multirow{2}{*}{2,205} & \multirow{2}{*}{0,137} \\
\hline & No & 338 & 75,4 & 48 & 10,7 & 386 & 86,2 & & \\
\hline \multirow{2}{*}{$\begin{array}{l}\text { Interpretación } \\
\text { radiográfica }\end{array}$} & Compleja & 8 & 1,8 & 2 & 0,4 & 10 & 2,2 & \multirow{2}{*}{0,384} & \multirow{2}{*}{0,534} \\
\hline & Sencilla & 380 & 84,8 & 58 & 12,9 & 438 & 97,8 & & \\
\hline
\end{tabular}

Tabla 4. Factores intraoperatorios asociados al resultado de la terapia

\begin{tabular}{|c|c|c|c|c|c|c|c|c|c|}
\hline \multirow{3}{*}{ Variable } & & \multicolumn{4}{|c|}{ Resultado } & \multirow{2}{*}{\multicolumn{2}{|c|}{ Total }} & \multirow{3}{*}{ Chi } & \multirow{3}{*}{$\mathbf{p}$} \\
\hline & & \multicolumn{2}{|c|}{ Éxito } & \multicolumn{2}{|c|}{ Fracaso } & & & & \\
\hline & & $\mathbf{n}$ & $\%$ & $\mathrm{n}$ & $\%$ & $\mathrm{n}$ & $\%$ & & \\
\hline \multirow{2}{*}{$\begin{array}{l}\text { Dolor durante el } \\
\text { tratamiento }\end{array}$} & Sí & 20 & 4,5 & 5 & 1,1 & 25 & 5,6 & \multirow{2}{*}{0,996} & \multirow{2}{*}{0,318} \\
\hline & No & 368 & 82,1 & 55 & 12,3 & 423 & 94,4 & & \\
\hline \multirow{2}{*}{ Acceso cameral } & Sí & 2 & 0,4 & 1 & 0,2 & 3 & 0,7 & \multirow{2}{*}{1,035} & \multirow{2}{*}{0,308} \\
\hline & No & 386 & 86,2 & 59 & 13,2 & 445 & 99,3 & & \\
\hline \multirow{2}{*}{$\begin{array}{l}\text { Adecuada calidad de } \\
\text { la obturación }\end{array}$} & Sí & 373 & 83,3 & 53 & 11,8 & 426 & 95,1 & \multirow{2}{*}{6,771} & \multirow{2}{*}{0,009} \\
\hline & No & 15 & 3,3 & 7 & 1,6 & 22 & 4,9 & & \\
\hline
\end{tabular}

Tabla 5. Otros factores asociados al resultado de la terapia

\begin{tabular}{|c|c|c|c|c|c|c|c|c|c|}
\hline \multirow{3}{*}{ Variable } & & \multicolumn{4}{|c|}{ Resultado } & \multirow{2}{*}{\multicolumn{2}{|c|}{ Total }} & \multirow{3}{*}{ Chi } & \multirow{3}{*}{$\mathrm{p}$} \\
\hline & & \multicolumn{2}{|c|}{ Éxito } & \multicolumn{2}{|c|}{ Fracaso } & & & & \\
\hline & & $\mathbf{n}$ & $\%$ & $\mathbf{n}$ & $\%$ & $\mathbf{n}$ & $\%$ & & \\
\hline \multirow{2}{*}{ Periodontitis crónica } & Sí & 0 & 0,0 & 1 & 0,2 & 1 & 0,2 & \multirow{2}{*}{6,481} & \multirow{2}{*}{0,010} \\
\hline & No & 388 & 86,6 & 59 & 13,2 & 447 & 99,8 & & \\
\hline \multirow{2}{*}{ Retratamiento } & Sí & 3 & 0,7 & 0 & 0,0 & 3 & 0,7 & \multirow{2}{*}{0,467} & \multirow{2}{*}{0,494} \\
\hline & No & 385 & 85,9 & 60 & 13,4 & 445 & 99,3 & & \\
\hline
\end{tabular}




\section{Discusión}

Los avances en la endodoncia han aumentado el porcentaje de éxito de la terapia ${ }^{12-14}$, el cual se encuentra entre el $70 \%$ y el $95 \%$ cuando el tratamiento es realizado por especialistas y entre el $64 \%$ y el $75 \%$, cuando es llevado a cabo por odontólogos generales según reportes de Velez et al..$^{15}$. Otros autores como Kherlakian et al. ${ }^{16}$ señalan tasas de éxito entre un $86 \%$ y un $98 \%$. Se observan de manera general datos en correspondencia con estos apuntes. En el presente trabajo fue usada la técnica estandarizada de Schilder para la preparación biomecánica de los canales, Friedman $e t$ al. ${ }^{2}$ en sus estudios al evaluar el $42 \%$ de dientes tratados mediante la técnica de Schilder y el $53 \%$ con la técnica de step-back no señalan diferencias significativas entre las tasas de éxito de los tratamientos realizados. Ellos concuerdan en que la reparación periapical y curación de la periodontitis apical está influenciada en gran medida por la presencia de microorganismos, su ambiente y la respuesta inmune del huésped y que la variación en los procedimientos no es el elemento esencial en la modificación de estos aspectos, si estos se guían de forma eficiente.

En contraste con el método empleado, la literatura actual recomienda el empleo de instrumental mecanizado mediante el uso de sistemas rotatorios, destacándose ventajas en relación a la simplicidad de la aplicación, rapidez, comodidad para el paciente, seguridad y sencillez en su uso. Informes sugieren resultados variables al ser comparadas las cifras de éxito de las mismas con técnicas convencionales y el empleo de limas manuales ${ }^{16}$.

Los resultados registrados en la investigación actual pueden ser considerados como satisfactorios, aunque no se desecha la preocupación por disminuir la incidencia de fracasos.

Se evidencia que el fracaso post tratamiento en la población estudiada estuvo relacionado con múltiples factores. Entre los referidos al paciente se destaca la edad como factor significativo. Se conoce que el envejecimiento condiciona el retraso de procesos reparativos debido a variaciones en el sistema circulatorio, cambios hísticos, la menor actividad de osteoblastos, osteoclastos entre otros aspectos. Varios estudios reconocen la importancia de este parámetro en el resultado del tratamiento y lo incluyen como posible predictor en el pronóstico de la terapia de canales, aunque no siempre alcanza valores significativos ${ }^{17,18}$ en desacuerdo con los resultados alcanzados en la presente investigación.

Dentro de los factores anatómicos, el estudio mostró a los dientes anteriores maxilares como los más comprometidos. Esto coincide con trabajos realizados por Fiallo et al. ${ }^{19}$. Ellos reportaron una mayor frecuencia de tratamientos llevados a cabo en los incisivos superiores $(66,6 \%)$. Mientras que el estudio de Monteza et al. ${ }^{20}$ informa la ejecución de la terapia en el 39,2\% de estos dientes. En la actual investigación una mayor frecuencia de tratamientos fueron emprendidos en dientes anteriores maxilares lo que coincide con los datos antes expuestos. Este particular puede ser explicado debido a la preocupación creciente de la población por patrones estéticos que conlleva a un mayor reclamo del servicio cuando se trata de la afectación de este grupo dentario, en función de lograr la preservación de la armonía facial. Otro elemento básico es la mayor accesibilidad de los incisivos para el operador lo que facilita la terapia y muchas veces condiciona el éxito del tratamiento. En la práctica odontológica actual aunque son de suma importancia aspectos relacionados con la fisiología del órgano dentario, priman criterios conservacionistas con relación al diente y el clínico deberá considerar durante la selección del caso la necesidad sentida del paciente, reconociendo posibles dificultades durante el procedimiento, en correspondencia con el grupo dentario en cuestión.

Además, se obtuvieron resultados relevantes en relación al tamaño del canal y la morfología radicular dentro de los aspectos anatómicos evaluados. Los resultados que se exponen coinciden con estudios previos que señalan mayor porcentaje de éxito en aquellos dientes que tienen uno o dos canales y mayor frecuencia de fracasos en molares de tres canales y una anatomía compleja y a veces impredecible $18,21-25$. Con respecto a la morfología de los canales es válido señalar que los canales atrésicos y sinuosos o aquellos dientes de curvatura pronunciada, donde en ocasiones no es posible llegar con el instrumental hasta la unión del cemento y la dentina en el conducto (cdc), afectan el resultado final del tratamiento al limitar la limpieza durante la preparación biomecánica. Otro elemento a considerar son los dientes de gran longitud (más de 25 milímetros) donde queda afectado el acceso directo de las limas al extremo más apical ${ }^{1,16,23,26}$. $\mathrm{Ng}$ et al. ${ }^{18}$ reportan que canales con extremo apical inaccesibles están asociados a bajas tasas de éxito. En contraste con la técnica empleada en esta investigación, estudios sugieren que se puede mejorar el acceso al canal con el empleo de técnicas corono-apicales por la posibilidad que las mismas generan en crear una mayor amplitud inicial en el extremo coronal que permite luego el paso más cómodo al extremo apical del canal. Se añade además el empleo del ácido dietil amino tetracético (EDTA) como garantía de una mayor permeabilidad en los canales ${ }^{16}$. $\mathrm{Ng}$ et al. ${ }^{18}$ al compilar datos sobre el tema concluyen que la destreza del profesional es un imperativo para alcanzar buenos resultados ante cualquier técnica empleada. La sensibilidad táctil, entrenamiento y experiencia en el uso de técnicas e instrumentos constituye un eslabón esencial para reducir la posibilidad de errores y complicaciones asociadas al fracaso. La determinación imprecisa de la longitud de trabajo en dientes de anatomía compleja puede favorecer la ocurrencia de accidentes endodónticos, como perforación apical y sobreobturación, las cuales son generalmente acompañadas de dolor postoperatorio. Por otra parte, el inicio de la reparación periapical puede prolongarse en el tiempo, aumentando así el número de fracasos por regeneración incompleta de los tejidos periapicales. Otro riesgo presente ante condiciones anatómicas peculiares, son la instrumentación incompleta y la obturación deficiente del canal radicular, con todos 
los problemas que ello trae (reagudización de la infección y de los síntomas, reinfección del canal radicular, aparición de lesiones apicales y dolor persistente debido a la inflamación de tejido pulpar no eliminado) ${ }^{27}$. Por otra parte Rodríguez-Niklitschek et al. ${ }^{21}$, así como otros autores ${ }^{22,23}$ sostienen la complejidad de la anatomía radicular como impedimento en la correcta desinfección del conducto.

En la presente investigación también se encontró una relevancia muy significativa de la sensibilidad pulpar en relación con el fracaso del tratamiento. Estos datos coinciden con el estudio realizado por Lin $e t a l^{7}$ donde se registró que el resultado final del tratamiento estuvo relacionado con el estado pulpar y periapical preoperatorio. La literatura sobre el tema sugiere que uno de los principales factores asociados con el fracaso endodóntico es la persistencia de la infección microbiana en el sistema de canales radiculares, generalmente ocasionado por la presencia de patologías periapicales en dientes con pulpas necróticas ${ }^{28}$.

La condición pulpo radicular previa conduce en ocasiones a fracasos endodónticos, esto puede deberse a que la enfermedad de origen endodóntico está directamente relacionada con la presencia de microorganismos en el sistema de canales que contribuyen a modificaciones de la zona periapical. Se conoce en este sentido que la presencia de canales accesorios aloja gran cantidad de contenido séptico y son muchas veces inaccesibles a los instrumentos endodónticos ${ }^{12,22,29}$. A nuestro juicio, se deberá velar siempre por una óptima apertura que permita el paso directo del instrumental al sistema de canales, así como por la selección apropiada de sustancias irrigadoras que limiten la infección ante estas situaciones específicas. Una prolija técnica de preparación biomecánica, en consonancia con el objetivo biológico de la conformación, permitirá obtener la permeabilidad dentinaria necesaria, para que las soluciones irrigantes actúen con mayor eficiencia 29-32. El empleo de hipoclorito de sodio con este fin suele tener buenos resultados lo cual se refleja a través del método empleado en este estudio. Reportes señalan el uso conjunto del hipoclorito de sodio y EDTA, cuyo efecto sinérgico ha sido comprobado, aunque estos datos no son consistentes con hallazgos microbiológicos y las tasas de éxito que se han alcanzado en los estudios donde se emplean son bajas (67\%), en contraste con el uso de hipoclorito de sodio al $0,5 \%$ (92\% de éxito) o hipoclorito de sodio al 5\% (86\% de éxito) lo cual es consistente con los resultados alcanzados. Investigaciones recientes describen el uso de hidróxido de calcio en unión de clorhexidina al $0,2 \%{ }^{18,30,31}$, basado en la posibilidad de acción eficiente contra el Enterococcus faecalis, sin embargo solo se señala un $62 \%$ de éxito con respecto a su empleo ${ }^{18}$.

Con respecto al nivel apical de la obturación la literatura menciona que el mejor pronóstico, se logra en los dientes en los que el material de relleno se halla entre 0 y 2 $\mathrm{mm}$ del ápice radiográfico, y se desecha la idea de la influencia marcada en el resultado final del tratamiento de diferentes materiales y técnicas para la obturación ${ }^{18}$. Es preciso añadir que la obturación de los canales radiculares ha mejorado en el tiempo, gracias a la introducción de nuevas tecnologías, que han contribuido a lograr un sellado más eficiente; los sistemas termoplastificados en la obturación radicular podrían ser una alternativa útil en la reducción de fallas con respecto al resultado del tratamiento aunque no siempre han logrado demostrar mayor eficacia clínica que los sistemas de obturación en frío, de ahí que un porcentaje importante de odontólogos sigue utilizando la técnica de compactación lateral en frío, método empleado en el presente estudio ${ }^{33}$. Otras investigaciones podrían ser guiadas para ahondar en este particular.

En el caso de sobre obturaciones disminuyen las tasas de éxito a un $76 \%$ y se constata en el caso de obturaciones de conducto cortas solo un $68 \%$ de éxito radiográfico y clínico ${ }^{34}$. El presente trabajo también mostró un resultado significativo en relación al factor calidad de la obturación con respecto a la evolución del tratamiento. Varios profesionales reportan que la sobre obturación tiene un efecto negativo en el pronóstico del tratamiento, estos hallazgos demuestran que el material de obturación puede actuar como cuerpo extraño causando irritación en el tejido periradicular ${ }^{18,25,35,36}$.

Los resultados con respecto a la calidad de la obturación del canal en la investigación realizada, sugieren la importancia que tiene el relleno eficiente; el cierre hermético después de una efectiva limpieza y conformación evita una nueva contaminación y por ende favorece la reparación de los tejidos ${ }^{18,34}$.

La presencia de periodontitis también arrojó un resultado significativo en relación al fracaso del tratamiento, lo cual coincide con apuntes de Ng YL et al. ${ }^{29}$ los que señalan, a partir de una revisión exhaustiva de la literatura, que el deterioro de la condición periodontal puede estar asociado a la recontaminación del diente. La relación entre la pulpa y el periodonto es dinámica. Los dientes que tienen pérdida parcial o completa del hueso de soporte pueden ser más susceptibles a enfermedades pulpares. La movilidad excesiva puede resultar en estenosis o desgarramiento de los vasos sanguíneos apicales. La invasión bacteriana ocurre a través del foramen apical y los conductos laterales, secundarios o cavointerradiculares expuestos por la enfermedad periodontal ${ }^{2,24,29}$.

Se puede establecer como conclusión del trabajo que existen diversos factores asociados a la evolución del tratamiento pulpo radicular, registrándose la edad, grupo de dientes, localización de los dientes, morfología radicular, el tamaño de los conductos, la sensibilidad pulpar, la calidad de la obturación y la enfermedad periodontal como los de mayor influencia en la muestra objeto de estudio. Estos hallazgos sugieren que estos factores pudieran ser usados con valor pronóstico (por ejemplo, mediante la construcción de un índice) en trabajos futuros para la predicción del resultado de la terapia pulpo radicular. 


\section{Referencias bibliográficas}

1. Toledo L, Alfonso M. Complejidad del tratamiento endodóntico, según factores asociados. Rev Cubana Estomatol [Internet]. 2016 [citado el 21 de marzo de 2017];53(2). Disponible en: http://scielo.sld.cu/scielo.php?script=sci_arttext\&pid=S0034-75072016000200002\&lng=es.

2. Friedman S, Abitbol S, Lawrence HP. Treatment outcome inendodontics: The Toronto Study-Phase 1: inicial treatment. J Endod. 2003;29(12):787-93.

3. Pedraza GR, Gandaria MD. Comportamiento de algunas enfermedades pulpares como urgencias en pacientes de 15 y más años. Multimed. 2013;17(4):1-8.

4. Toledo L, Alfonso M. Consideraciones en relación con la complejidad del tratamiento endodóntico. Medicentro Electrónica [Internet]. 2015 [citado el 21 de marzo de 2017];19(2). Disponible en: http://scielo.sld.cu/scielo.php?script=sci_arttext\&pi$\mathrm{d}=$ S1029-30432015000200007\&lng=es.

5. Field JW, Gutmann JL, Solomon ES, Rakusin H. A clinical radiographic retrospective assessment of the success rate of single-visit root canal treatment. Int Endod J. 2004;37(1):70-82.

6. Toledo L, Alfonso M, Barreto E. Evolución del tratamiento endodóntico y factores asociados al fracaso de la terapia. Medicent Electrón [internet]. 2016 [citado el 14 de enero de 2017];20(3). Disponible en: http://scielo. sld.cu/scielo.php?pid=S1029-30432016000300006\&script=sci_arttext\&tlng=en

7. Lin C, Xu L, Chen YX, Liang Y, Chen XL, Lin Y, et al. A statistical model for predicting the retrieval rate of separated instruments and clinical decision-making. J Dent Sci. 2015;10(4):423-30.

8. AAE: Endodontic Case Difficulty Assessment Form and Guidelines. [Internet]. Chicago: American Association of Endodontists; Jul 1, 2006 [citado 26 de mayo 2017]. Disponible en: http://www.aae.org/uploadedfiles/dental_professionals/endodontic_case_assessment/2006casedifficultyassessmentformb_edited2010.pdf

9. Schneider SA. Comparison of canal preparations in straight and curved root canals. Oral Surg Oral Med Oral Pathol. 1971;32(2):271-75.

10. Gutmann JL. Clinical, radiographic, and histologic perspectives on success and failure in endodontics. Dent Clin North Am. 1992;36(2):379-92.

11. Miller DA. Significant and highly significant. Nature. 1966; 210(5041):1190.

12. Estrela C, Holland R, Estrela CR, Alencar AH, Sousa-Neto MD, Pécora JD. Characterization of successful root canal treatment. Braz Dent J. 2014;25(1):3-11.

13. Friedman S, Mor C. The success of endodontic therapy healing and functionality. J Calif Dent Assoc. 2004;32(6):493-503.

14. Borén DL, Jonasson P, Kvist T. Long-term survival of endodontically treated teeth at a public dental specialist clinic. J Endod. 2015;41(2):176-81.

15. Velez EP, Cardona A. Factores asociados a la supervivencia del diente con endodoncia en pacientes mayores de 20 años, atendidos en una IPS privada en el periodo 2006 a 2012. Rev Fac Odontol Univ Antioq. 2014;25(2):283-98.

16. Kherlakian D, Cunha RS, Ehrhardt IC, Zuolo ML, Kishen A, da Silveira Bueno CE. Comparison of the incidence of postoperative pain after using 2 reciprocating systems and a continuous rotary system: a prospective randomized clinical trial. J Endod. 2016;42(2):171-6.

17. Arias A, la Macorra JC, Hidalgo JJ, Azabal M. Predictive models of pain following root canal treatment: a prospective clinical study. Int Endod J. 2013;46(8):784-93.

18. Ng YL, Mann V, Gulabivala K. A prospective study of the factors affecting outcomes of non-surgical root canal treatment: part 2: tooth survival. Int Endod J. 2011;44(7):610-25.

19. Fiallo CJ, Báez FA, Reyes VO. Fracasos del tratamiento endodóntico en pacientes atendidos en el servicio de urgencias estomatológicas Endodontic treatment failures in patients treated in the emergency department of stomatology. Medimay. 2014;20(2):219-230.

20. Monteza T. Prevalencia de tratamientos de conductos radiculares en piezas dentarias permanentes en la clínica estomatológica [tesis de licenciatura]. [Chiclayo]: Facultad de Ciencias de la Salud, Universidad Señor de Sipán; 2016. 90 p.

21. Rodríguez-Niklitschek C, Oporto GH. Determinación de la longitud de trabajo en endodoncia: Implicancias clínicas de la anatomía radicular y del sistema de canales radiculares. Int J Odontostomat. 2014;8(2):177-83.

22. Abella F, Patel S, Durán-Sindreu F, Mercadé M, Roig M. Mandibular first molars with disto-lingual roots: review and clinical management. Int Endod J. 2012;45(11):96378 .

23. Naseri M, Safi Y, Baghban AA, Khayat A, Eftekhar L. Survey of anatomy and root canal morphology of maxillary first molars regarding age and gender in an Iranian population using cone-beam computed tomography. Iran Endod J. 2016;11(4):298-303.

24. Lee CB, Chang YH, Wen PC, Li CY. Association of Failed Root Canal Treatment with Dentist and Institutional Volumes: A Population-based Cohort Study in Taiwan. J Endod. 2017;43(10):1628-34.

25. Monardes-Cortés H, Abarca-Reveco J, Chaparro-González D, Pizarro-Gamboa F. Hallazgos radiográficos de connotación endodóntica utilizando tomografía computarizada de haz cónico. Av Odontoestomatol. 2015;31(2):59-65.

26. Kqiku L, Städtler P. Radiographic versus electronic root canal working length determination. Indian J Dent Res. 2011;22(6):777-80.

27. Somma F, Castagnola R, Lajolo C, Paternò-Holtzman L, Marigo L. In vivo accuracy of three electronic root canal length measurement devices: Dentaport ZX, Raypex 5 and ProPex II. Int Endod J. 2012;45(6):552-6.

28. Rodríguez-Niklitschek C, Oporto GH. Implicancias clínicas de la contaminación microbiana por Enterococcus faecalis en canales radiculares de dientes desvitalizados: Revisión de la literatura. Rev Odontol Mex. 2015;19(3):181-86. 
29. Ng YL, Mann V, Rahbaran S, Lewsey J, Gulabivala K. Outcome of primary root canal treatment: systematic review of the literature-Part 2. Influence of clinical factors. Int Endod J. 2008;41(1):26-31.

30. Ferraz CC, de Almeida Gomes BP, Zaia AA, Teixeira FB, de Souza-Filho FJ. In vitro assessment of the antimicrobial action and the mechanical ability of chlorhexidine gel as an endodontic irrigant. . J Endod. 2001;27(7):452-5.

31. Menakaya IN, Oderinu OH, Adegbulugbe IC, Shaba OP. Incidence of postoperative pain after use of calcium hydroxide mixed with normal saline or $0.2 \%$ chlorhexidine digluconate as intracanal medicament in the treatment of apical periodontitis. Saudi Dent J. 2015;27(4):187-93.

32. Gutmann JL, Lovdahl PE. Solución de problemas en el dolor no odontógeno. En: Solución de problemas en Endodoncia. Prevención, identificación y tratamiento. 5ta. ed. Barcelona:Elsevier S.L.;2012.
33. Aracena D, Bustos L, Alcántara R, Aguilera O, Aracena A, Luengo P. Comparación de la Calidad de Obturación Radicular, entre el Sistema Termoplastificado Calamus y el Sistema de Compactación Lateral en Frío. Int J Odontostomat. 2012;6(2):115-21.

34. Sjögren ULF, Hägglund B, Sundqvist G, Wing $\mathrm{K}$. Factors affecting the long-term results of endodontic treatment. Journal of endodontics. J Endod.1990;16(10):498-504.

35. Shinbori N, Grama AM, Patel Y, Woodmansey K, He J. Clinical outcome of endodontic microsurgery that uses Endo Sequence BC root repair material as the root-end filling material. J Endod. 2015;41(5):607-12.

36. Imura N, Pinheiro ET, Gomes BP, Zaia AA, Ferraz CC, Souza-Filho FJ. The outcome of endodontic treatment: a retrospective study of 2000 cases performed by a specialist. J Endod. 2007;33(11):1278-82. 
